\title{
Distribution of magnetic shear angle in an emerging flux region
}

\author{
Sanjay Gosain \\ Udaipur Solar Observatory, Physical Research Laboratory, \\ P. Box No. 198, Udaipur 313001, Rajasthan, India \\ email: sgosain@prl.res.in
}

\begin{abstract}
We study the distribution of magnetic shear in an emerging flux region using the high-resolution Hinode/SOT SP observations. The distribution of mean magnetic shear angle across the active region shows large values near region of flux emergence i.e., in the middle of existing bipolar region and decreases while approaching the periphery of the active region.
\end{abstract}

Keywords. Sun: active regions, sunspot: flux emergence

\section{Introduction}

The non-potentiality of the magnetic field in solar active regions provides the freeenergy needed to fuel the energetic events like solar flares and CMEs. The non-potentiality may result either due to the shearing motions of the footpoint or due to the emergence of pre-stressed magnetic flux from the convection zone into the photosphere. The study of magnetic flux emergence in active regions is therefore very important for understanding the flaring activity. The high-quality vector magnetograms derived from spectropolarimetric (SP) observations by space-based Hinode Solar Optical Telescope (SOT) (Tsuneta et al. 2008) are very useful for such studies. Although, the cadence of such SP observations is not fast enough to capture the dynamic evolution of magnetic field. One can, however, study the spatial distribution of magnetic non-potentiality in such active regions.

One of the important parameters used to characterize the non-potentiality of the magnetic field is the magnetic shear angle (Hagyard et al. 1984). The magnetic shear angle $\Delta \psi$ is defined as the angle between the azimuths of the observed and the potential field i.e., $\Delta \psi=\psi_{o}-\psi_{p}$. The larger is the magnetic shear angle (henceforth MSA) more non-potential is the magnetic field.

The distribution of MSA in an active region, which is going through flux emergence process, is useful in order to understand the buildup of non-potentiality in active regions. In the present paper we present results from such a study.

\section{Observations and analysis methods}

The magnetic flux was emerging in the middle of an existing bipolar region NOAA 10978, during 13 December 2007. Several thin elongated fibril structures were seen in the region of flux emergence. These emerging flux tubes displace the photospheric plasma, causing an appearance of diverging flow pattern. As the flux emergence progresses the 

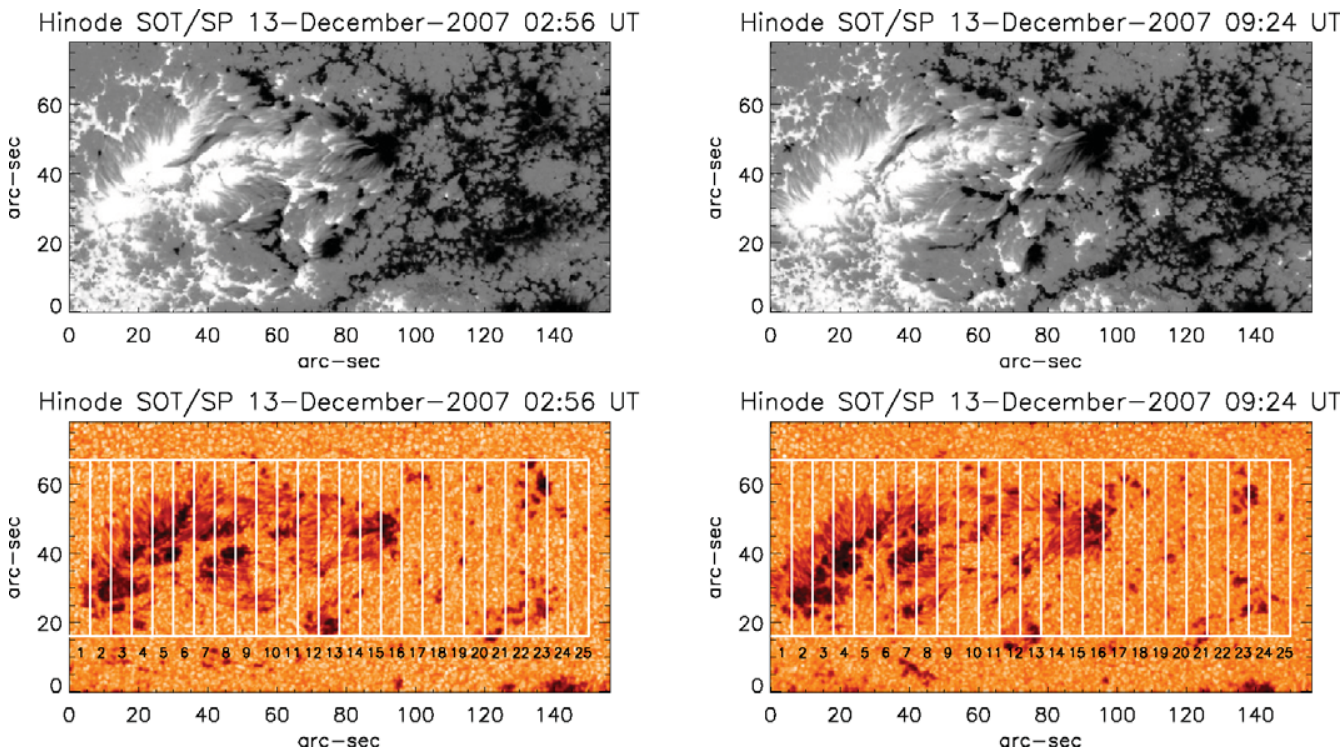

Figure 1. The top panels show the longitudinal field map of the emerging flux region in NOAA 10978 during 13 December 2007. The bottom panels show the continuum intensity maps of this region. The distribution of magnetic shear across the region is estimated by calculating the mean shear angle inside rectangular boxes labeled 1 through 25 . The variation of magnetic shear angle across the region from boxes 1 to 25 is shown in figure 2 .

foot points of the fibrils move apart and become more and more vertical and bundle together to form sunspots.

The spectropolarimetric observations of this active region were obtained by the spectropolarimeter instrument (Ichimoto et al. 2008) onboard Hinode space mission (Kosugi et al. 2007). The magnetic field vector was derived by using a Stokes inversion code called MERLIN (Lites et al. 2007). The code performs a non-linear least squares fit of the observed Stokes profiles with theoretical Stokes profiles computed under the MilneEddington model atmosphere assumptions.

The top panel of Figure 1 shows the longitudinal magnetic field map of this active region at two different stages during its evolution. The small bipoles near the polarity inversion line (PIL), with opposite polarity orientation as compared to the overall active region polarity suggests the presence of undulatory U-loops. The bottom panel of Figure 1 shows the continuum intensity maps of the active region. The typical features of an emerging flux region, like elongated thin fibril like structures, can be clearly noticed. We evaluate the variation of shear angle across the active region by computing the mean shear angle within each of the rectangular box labeled 1-25. The boxes are distributed along the bipolar axis of the active region so as to get spatial profile of shear across the active region. We remove the tilt of the active region by rotating the map so that the bipolar axis is horizontal. The bipolar axis is computed by joining the centroid of the longitudinal flux in either polarity of the active region. The shear angle is computed for each pixel of the map and is averaged inside each of the box $1-25$. The plot in Figure 2 shows the distribution of mean magnetic shear angle across the active region. 

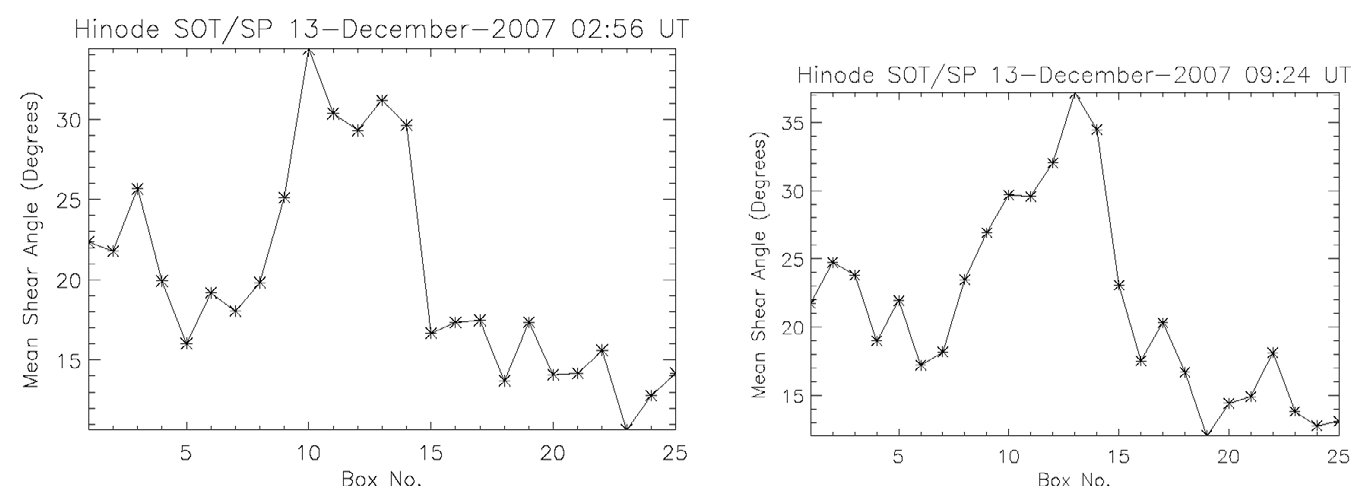

Figure 2. The left and right panels show the distribution of magnetic shear angle across the active region at two different times during 13 December 2007. The abscissa shows the box number and the ordinate shows the mean shear angle inside the corresponding box.

\section{Results and discussions}

It can be seen from Figure 2 that the mean shear angle is not uniformly distributed across the active region. The mean shear angle is higher in the central portion of the active region where the flux tubes emerge. The peak value of the shear angle in the central portion is about 30-35 degrees, while at the periphery it is about 25 degrees. The pattern persists during the two time intervals which are separated by about six hours as shown in Figure 1.

These observations show that the magnetic field in the middle portion of the emerging flux region consists of the largest amount of non-potentiality. The present results obtained with the high-resolution and high-sensitive polarimetry put shear distribution studies on a firm footing. Similar results were obtained by Schmieder et al. (1996) for NOAA 6718 where the comparison of coronal field structure with linear force-free field computation yielded a differential magnetic field shear model. The present observations support their argument that the decreased shear in the outer portions of the active region is probably due to continual relaxation of the magnetic field to lower energy state in the older portions of the active region. In future we plan to carry out a similar analysis of magnetic shear in decaying or dormant active regions to see how different the pattern appears as compared to the emerging flux regions.

\section{Acknowledgements}

The presentation of this paper in the IAU Symposium 273 was possible due to financial support from the National Science Foundation grant numbers ATM 0548260, AST 0968672 and NASA - Living With a Star grant number 09-LWSTRT09-0039. Hinode is a Japanese mission developed and launched by ISAS/JAXA, with NAOJ as domestic partner and NASA and STFC (UK) as international partners. It is operated by these agencies in co-operation with ESA and NSC (Norway).

\section{References}

Hagyard, M. J., Teuber, D., West, E. A., \& Smith, J. B. 1984, Solar Phys., 91, 115

Ichimoto, K., et al. 2008, Solar Phys., 249, 233 
Kosugi, T., et al. 2007, Solar Phys., 243, 3

Lites, B., Casini, R., Garcia, J., \& Socas-Navarro, H. 2007, Solar Phys., 78, 148.

Schmieder, B., Demoulin, P., Aulanier, G., \& Golub, L. 1996, Astrophys. J., 467, 881

Tsuneta, S., et al. 2008, Solar Phys., 249, 167 